\title{
Developing a framework for assessing muscle effort and postures during computer work in the field: the effect of computer activities on neck/shoulder muscle effort and postures
}

\author{
Bruno Garza J.L. ${ }^{a}$, Eijckelhof B.H.W. ${ }^{\text {b,c }}$, Johnson P.W. ${ }^{\text {, }}$, Raina S.M. ${ }^{\mathrm{a}}$, Rynell, P. ${ }^{\mathrm{f}}$, Huysmans, M.A. ${ }^{\mathrm{b}, \mathrm{c}}$, \\ van Dieën J.H. ${ }^{\mathrm{c}, \mathrm{d}}$, van der Beek A.J., ${ }^{\mathrm{b}, \mathrm{c}}$, Blatter B.M. ${ }^{\mathrm{c}, \mathrm{e}}$ and Dennerlein J.T. ${ }^{\mathrm{a}, \mathrm{g}^{*}}$ \\ ${ }^{a}$ Department of Environmental Health, Harvard School of Public Health, 665 Huntington Avenue, Boston, MA, \\ 02115, USA \\ ${ }^{\mathrm{b}}$ Department of Public and Occupational Health and the EMGO Institute for Health and Care Research, VU \\ University Medical Center, Van der Boechorststraat 9, 1081 BT, Amsterdam, The Netherlands \\ ${ }^{c}$ Body@Work Research Center on Physical Activity, Work and Health, TNO-VU/VUmc, Van der Boechorststraat \\ 9, 1081 BT, Amsterdam, The Netherlands \\ ${ }^{d}$ Research Institute MOVE, Faculty of Human Movement Sciences, VU University, Van der Boechorststraat 9 , \\ 1081 BT, Amsterdam, The Netherlands \\ ${ }^{e}$ Netherlands Organisation for Applied Scientific Research, TNO, Polarisavenue 151, 2132 JJ, Hoofddorp, The \\ Netherlands \\ ${ }^{f}$ Department of Environmental and Occupational Health Sciences, Box 357234, Seattle, WA, 98195, USA \\ ${ }^{g}$ Department of Orthopaedic Surgery, Brigham and Women's Hospital, Harvard Medical School, 75 Francis \\ Street, Boston, MA, 02115 U.S.A.
}

\begin{abstract}
The present study, a part of the PROOF (PRedicting Occupational biomechanics in OFfice workers) study, aimed to determine whether trapezius muscle effort was different across computer activities in a field study of computer workers, and also investigated whether head and shoulder postures were different across computer activities. One hundred twenty participants were measured continuously for two hours each while performing their own computer work. Keyboard activities were associated with the highest intensity of left and right trapezius muscle efforts, and mouse activities were associated with the smallest variability in left and right trapezius muscle efforts. Corresponding trends in head and shoulder postures included that the greatest head flexion and left and right shoulder internal rotation was observed during keyboard activities, and that the smallest variability in head flexion, head lateral tilt, and right shoulder internal rotation was observed during mouse activities. Identifying which muscle efforts and postures are different across computer activities is the first essential step for developing prediction rules for muscle efforts and postures, which can be used to link muscle efforts and postures to musculoskeletal symptoms in epidemiological studies.
\end{abstract}

Keywords: office work, exposure assessment, upper extremity, task, trapezius

${ }^{*}$ Corresponding author. E-mail: jax@hsph.harvard.edu. 


\section{Introduction}

Office workers, who spend many hours per day working with a computer, often experience musculoskeletal symptoms in their neck and shoulder regions [1]. The one-year prevalence of neck and shoulder symptoms among office workers has been estimated to be between $24 \%$ and $44 \%$ based on several large-scale studies [2, 3, 4].

One generic risk factor for musculoskeletal symptoms that has been identified is prolonged periods of sustained muscle effort with little variation in exposure $[5,6]$. During computer work, these prolonged periods of activation have mainly been identified in the trapezius muscle, [7] which is also the site at which neck and shoulder musculoskeletal symptoms frequently occur [8].

Although computer use is associated with increased intensity and decreased variability of trapezius muscle effort, the type of computer activity performed by the computer worker may influence the intensity and variability experienced. In a laboratory study where trapezius muscle effort was measured during simulated computer work tasks composed of varying amounts of keyboard, mouse, and idle activities, differences in the intensity of trapezius muscle effort were observed across those tasks [9]. However, in this study the variability of trapezius muscle effort was not examined. Moreover, the study was performed in the laboratory, and did not examine differences across computer activities directly. It is unclear whether this relationship exists in the field, where other sources of variability such as workstation setup and type of work performed may influence the muscle efforts.

To address these concerns, a cross-sectional field study was performed in which muscle effort, head, and shoulder postures as well as computer activities of 120 office workers were measured directly and continuously while they performed their normal work at their own workstations [10]. The study's hypothesis was that trapezius muscle effort will be different across computer activities in field measurements of computer workers. This study also investigated whether there were differences in head and shoulder postures across activities, which may explain the differences in trapezius muscle effort.

\section{Methods}

Data for this study came from the larger PROOF study (PRedicting Occupational biomechanics in OFfice workers); a cross-sectional field study in which electromyography (EMG) and postural data were collected on a sample of 120 computer workers. All participants used the mouse with their right hand during measurements. While participants worked at their actual computer workstation, two wireless direct measurement sensors collected muscle activity from the right and left trapezius and three wireless inclinometers collected postures from the right and left shoulder and head [10]. Computer interaction monitoring software recorded computer use. These data provided the basis to parse the EMG and postural data into respective computer activities (keyboard activities, mouse activities, and idle activities $<30 \mathrm{~s}$ ) [11]. Summary statistics calculated included the $10^{\text {th }}, 50^{\text {th }}$ and $90^{\text {th }}$ percentiles of the muscle effort and posture signals and the difference between the $90^{\text {th }}$ and $10^{\text {th }}$ percentiles (a measure of variability). One-way repeated-measures analyses of variance (ANOVAs) were used to determine whether each muscle effort, head, and shoulder posture varied across activities. Post-hoc analyses used t-tests with Bonferroni correction for multiple comparisons to test for differences between each computer activity.

\section{Results}

Both the left and right trapezius muscle effort significantly differed across keyboard, mouse, and idle activities $(p<0.001)$. The highest muscle efforts for the $10^{\text {th }}, 50^{\text {th }}$, and $90^{\text {th }}$ percentiles of the right trapezius, and for the $10^{\text {th }}$ and $50^{\text {th }}$ percentiles of the left trapezius were observed during keyboard activities. The smallest variability in left and right trapezius muscle efforts were observed during mouse activities (Figure 1).

The head posture demonstrated similar trends corresponding to the results for trapezius muscle effort. The greatest head flexion values were observed during keyboard activities. The smallest variability in head flexion and lateral tilt were observed during mouse activities (Figure 2).

The right and left shoulder rotation angles also demonstrated similar trends corresponding to the trapezius muscle effort results. The greatest left and right shoulder internal rotation values were observed during keyboard activities $(\mathrm{p}<0.001)$. The smallest 
variability in right shoulder internal rotation was observed during mouse activities (Figure 3).

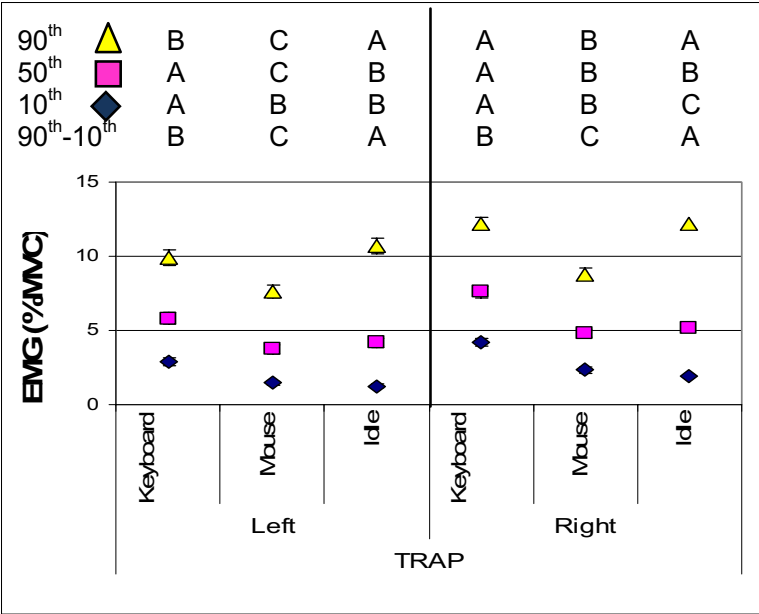

Fig. 1. Mean (standard error) $10^{\text {th }}$ (diamond), $50^{\text {th }}$ (square), and $90^{\text {th }}$ (diamond) percentiles of muscle effort expressed as a \%MVC averaged across all participants $[n=118]$. Letters $(A, B, C)$ indicate significant differences across computer activities. Different letters indicate which computer activities were different from one another. " $\mathrm{A}$ " represents the highest muscle effort values for each muscle effort parameter and muscle. When no significant differences were found, it is indicated by p-values greater than 0.05 .

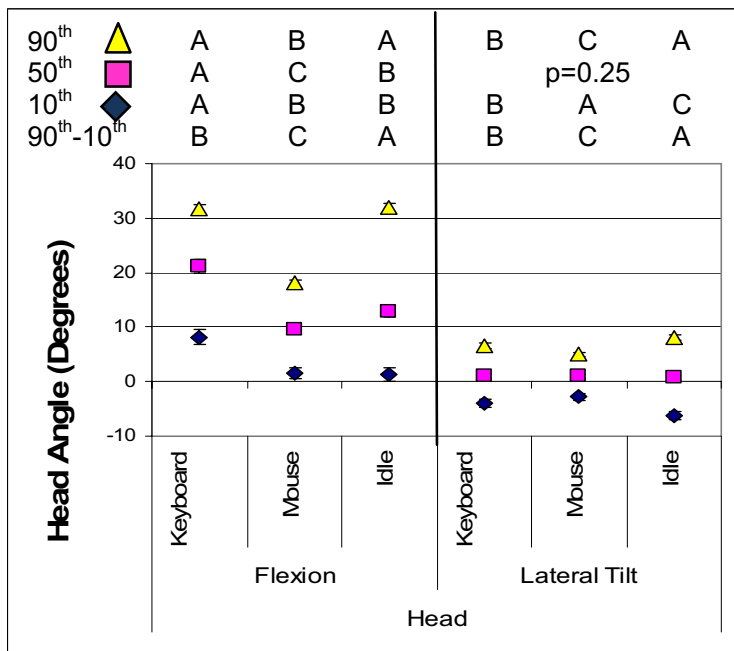

Fig. 2. Mean (standard error) $10^{\text {th }}$ (diamond), $50^{\text {th }}$ (square), and $90^{\text {th }}$ (diamond) percentiles of head postures averaged across all participants [ $n=118]$. Letters (A, B, C) indicate significant differences across computer activities. Different letters indicate which computer activities were different from one another. "A" represents the highest values for each parameter. When no significant differences were found, it is indicated by p-values greater than 0.05 .

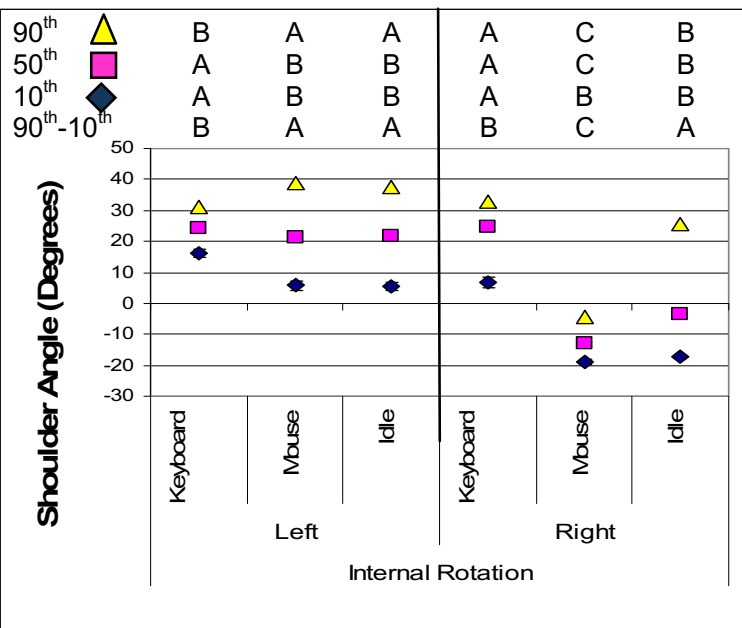

Fig. 3. Mean (standard error) $10^{\text {th }}$ (diamond), $50^{\text {th }}$ (square), and $90^{\text {th }}$ (diamond) percentiles of shoulder rotation postures averaged across all participants $[n=118]$. Letters (A, B, C) indicate significant differences across computer activities. Different letters indicate which computer activities were different from one another. " $A$ " represents the highest values for each shoulder angle posture. When no significant differences were found, it is indicated by $\mathrm{p}$-values greater than 0.05 .

\section{Discussion}

The goal of this study was to determine whether measures of trapezius muscle effort, head, and shoulder postures differed across computer activities. This study reported differences in trapezius muscle effort across computer activities, with the highest muscle efforts observed during keyboard activities and the smallest variability in muscle efforts observed during mouse activities. Although previous studies have reported that computer work can lead to increased trapezius muscle effort intensity and decreased variability, which may in turn lead to the development of MSDs, it appears that this effect may be influenced by the type of computer activity that a worker is performing.

The observations reported here could be used to inform interventions aiming to modify trapezius muscle effort exposures. Since muscle effort ampli- 
tude and variability are different across different computer tasks, the composition of computer tasks could be changed to alter the overall muscle effort amplitude and variability experienced by users during computer work. This may be done using computer shortcuts, which would increase mouse activities while decreasing keyboard activities.

We found some trends in head and shoulder postures that corresponded to the observations for the trapezius muscle effort trends. The greatest head flexion and left and right shoulder internal rotation were observed during mouse activities and the smallest variability in head flexion, head lateral tilt, and right shoulder internal rotation were observed during keyboard activities. The increased trapezius muscle effort intensity observed during mouse activities and decreased trapezius muscle effort variability observed during keyboard activities may be influenced by the head and shoulder rotation postures assumed during each activity. Thus, these postures could be the target for interventions aimed at reducing loading of the trapezius activation levels that in turn may reduce MSDs among computer workers.

Identifying which muscle efforts and postures are different across computer activities is the first essential step for developing prediction rules for muscle efforts and postures. These prediction rules could be used to predict the muscle efforts and postures in a larger cohort of office workers for whom only computer activity data is collected (through computer interaction monitoring software). Prediction rules are a non-invasive, simple, and cost effective way to generate muscle efforts and postures for computer workers. The predicted muscle efforts and postures could be used in epidemiological studies investigating the relationship between postures and muscle efforts experienced during computer use and musculoskeletal symptom development.

\section{References}

[1] NSR/IOM NRCaloM. Musculoskeletal Disorders and the workplaces: Low Back and Upper Extremities. National Academy Press, Washington, D.C. (2001).

[2] J.H. Andersen, A. Kaergaard, S. Mikkelsen, U.F. Jensen, P. Frost, J.P. Bonde, N. Fallentin and J.F. Thomsen, Risk factors in the onset of neck/shoulder pain in a prospective study of workers in industrial and service companies, Occupational and Environmental Medicine 60(9) (2003), 649654.

[3] B. Juul-Kristensen, K. Søgaard, J. Strøyer and U.F. Jensen, Computer users' risk factors for developing shoulder, elbow and back symptoms, Scandinavian Journal of Work and Environmental Health 30(5) (2004), 390-398.

[4] L.P. Brandt, J.H. Andersen, C.F. Lassen, A. Kryger, E. Overgaard, I. Vilstrup and S. Mikkelsen, Neck and shoulder symptoms and disorders among Danish computer workers, Scandinavian Journal of Work and Environmental Health 30(5) (2004), 399-409.

[5] J. Wahlström, Ergonomics, musculoskeletal disorders and computer work, Occupational Medicine 55(3) (2005), 168176.

[6] S.E. Mathiassen, Diversity and variation in biomechanical exposure: what is it, and why would we like to know?, Applied Ergonomics 37(4) (2006), 419-427.

[7] J.M. Richter, S.E. Mathiassen, H.P. Slijper, E.A. Over and M.A. Frens, Differences in muscle load between computer and non-computer work among office workers, Ergonomics 52(12) 2009, 1540-1555.

[8] C. Jensen, K. Nilsen, K. Hansen and R.H. Westgaard, Trapezius muscle load as a risk indicator for occupational shoulder-neck complaints, International Archives of Occupational and Environmental Health 64(6) (1993), 415-423.

[9] J.T. Dennerlein and P.W. Johnson, Different Computer tasks affect the exposure of the upper extremity to biomechanical risk factors, Ergonomics 49 (2006), 45-61.

[10] B.H.W. Eijckelhof, M.A. Huysmans, B.M. Blatter, J.L. Bruno, P.W. Johnson, A.J. van der Beek, J.H. van Dieen, and J.T. Dennerlein, Interactions of biomechanics and psychosocial stressors in relation to development of MSDs in the modern office: The 'PROOF' study protocol. Poster session presented at the 7th International Scientific Conference on Prevention of Work-Related Musculoskeletal Disorders, Angers, France. (August, 2010).

[11] C.H. Chang, P.W. Johnson and J.T. Dennerlein, A wide range of activity duration cutoffs provided unbiased estimates of exposure to computer use, Journal of Occupational and Environmental Hygiene 5 (2008), 790-796. 